\title{
Heterogeneous architecture for reversible watermarking system for medical images using Integer transform based Reverse Contrast Mapping
}

\author{
Subodh S. Ingaleshwar ${ }^{\text {a\# }}$, D. Jayadevappa ${ }^{\mathrm{b}}$, Nagaraj V. Dharwadkar ${ }^{\mathrm{c}}$ \\ ${ }^{a}$ Research Scholar, Dept. of E \& IE, JSSATE, Bengaluru, India \\ e-mail: subodh.ingleshwar@gmail.com \\ ${ }^{b}$ Dept. of E \& IE, JSS Academy of Technical Education, Bengaluru \\ e-mail: devappa22@gmail.com \\ 'Rajarambapu Institute of Technology, Affiliated to SUK, Sangli, India \\ e-mail: nagaraj.dharwadkar@ritindia.edu \\ \# Corresponding author
}

Article History:Received:11 november 2020; Accepted: 27 December 2020; Published online: 05 April 2021

\begin{abstract}
Algorithms in multimedia security including machine learning and deep learning find its way into more and more application field resulting in strict power and area constraints. This challenges traditional homogeneous computing concepts and drives the development of new, heterogeneous architectures. One way to attain a balance of high data throughput and flexibility is to combine soft-core FPGA accelerators with CPUs as hosts. In this paper, in order to achieve higher performance, low latency, unlimited re-configurability and most important very high energy efficiency, we have proposed a hybrid architecture using CPU and FPGA. These components are then dynamically configured to form complex algorithms directly in hardware by implementing reversible watermarking of medical images. We are proposing such reversible watermarking using Integer transform based Reversible Contrast Mapping (RCM) algorithm. We got improvement in results with respect to Peak Signal to Noise Ratio (PSNR) and Structural Similarity (SSIM), when compare to the implementation in proposed architecture and simulation results of different image formats like jpeg, png and DICOM.
\end{abstract}

Keywords: Heterogeneous, Reversible Watermarking, Medical Images, Field Programmable Gate Array, Central Processing Unit, Reverse Contrast Mapping, Peak Signal to Noise Ratio, Structural Similarity.

\section{Introduction}

Transmission of medical information through networks leads to security issues. In medical image watermarking, electronic patient record (EPR) is embedded in an image without any considerable changes. With the significant increase in the transaction of medical images via internet and other mobile application platform, there is a security concern of illegal use of medical information. Thus, security of medical information is a primary requirement with respect to related attributes such as Confidentiality, Reliability (Integrity, Authenticity) and Availability. In the computation of the requirements of medical image watermarking like Imperceptibility, Robustness (Resilient against attacks), Payload Capacity (Larger the capacity of watermark, greater the compromise with fidelity of image) Reversibility and Cost effective (computational cost), a proper tradeoff is required to attain an optimized solution. Integer transform based Reversible image watermarking method described by Coltuc et al., [1, 2, 3, 4, 5, 6] accomplishes different transform applied on pair of pixels. Unique feature of this transform is during data embedding; even if the LSB of the transformed pixels are lost then also the bits can be retrieved. Reversible contrast mapping offers high data embedding at low visual distortion with very low computational complexity.

The constituents of the paper are as follows. The section 2 describes the work done in spatial domain based computations and advantages of combining Image processing, FPGA \& Hardware Description Language (HDL). Section 3 represents the proposed scheme in embedding watermark in medical image. Section 4 compares the FPGA Implementation of RCM with comparing earlier results.

\section{Related work}

Few reversible watermarking techniques are listed below, based on pixels of one or two randomly selected subsets of an image.

a) Least Significant Bit Coding (LSB):

The LSB, in the bitwise representation of the target image replaced with the watermarking bit.

*Corresponding author: Subodh S. Ingaleshwar 
b) Predictive coding scheme by Matsui and Tanaka [7] is more robust compare to LSB technique, where the correlations between adjacent pixels are extracted.

c) Correlation-Based Techniques: Watermarking detection mechanism uses a pseudo random noise added to an image.

Although these techniques are exert low computational cost. But these are not dependable when subjected to operations such as filtering and compression.

Let us consider some technical excerpts from spatial domain operations like Data compression [8,9], Histogram modification [10, 11, 12, 13], Reverse Contrast Mapping , Difference Expansion $[14,15,16,17]$, Prediction Error Adjustment [18,19] for comparison. Among them RCM is quite popular because of its low computational cost.

Based on Spatial domain, many real time analyses of reversible watermarking algorithms on medical images have been proposed by various researchers.

a) Maity H K et.al.[19]: Verification of authenticity of medical images.

b) Zain et.al. [20] : Verification of the integrity and authenticity of medical images.

c) X. Guo et.al. [21]: Based on difference expansion based on the calculations of adjacent pixel values.

d) M. Kundu et.al. [22]: EPR data is embedded in RONI using advanced some encryption standard.

e) Saraju p. mohanty et.al [23] presented a paper "VLSI implementation of invisible digital watermarking algorithms towards the development of a secure jpeg encoder". In this paper, author has developed a hardware system which can insert both robust and fragile invisible watermark. Hardware emulation has more significance over software simulation in the analysis of low power, high performance and reliability.

f) Hirak kumar et.al, [24, 25] showed RCM-RM is applied on pair of pixels \& LSB is applied to embed secret information. Security of hidden data is maintained in this paper. Two architectures one for block size $(8 \times 8)$ and other for block size $(32 \times 32)$ are developed. 6 stage pipelining is allowed in the architecture. Proposed real time implementation with low cost and simplification of hardware accessibility is addressed.

But flexibility in terms of hardware accessibility is quite challenging in dedicated FPGA circuitry. Hence a heterogeneous architecture has been proposed using same algorithms of Reverse Contras Mapping. The results for different formats of images are compared and with initial implementation we can see minor improvement in the results when compare to simulation results.

\section{Proposed Work}

Fig.1 shows the block diagram of proposed Heterogeneous Architecture.

The purpose of this work is to describe a heterogeneous architecture for Medical Image Analysis using Reversible Watermarking. Proposed block diagram is divided into, Central Processing Unit Operations (CPU) and FPGA accelerator implementations (FPGA). Table 1 describes the functions and target device for performing the operation.

\begin{tabular}{|l|l|c|}
\hline \multicolumn{1}{|c|}{ Functions } & \multicolumn{1}{|c|}{ Specifications } & Target \\
\hline Storage of medical image & $256^{*} 256$ image of different formats & CPU \\
\hline Pre-processing & $\begin{array}{l}\text { Get file, resize, typecasting and } \\
\text { concatenation, EPR using GUI. }\end{array}$ & CPU \\
\hline Segmentation & 4 point selection mechanism & CPU \\
\hline Addition of security key & 16 bit digit used as security key & FPGA \\
\hline Watermarking using EPR & EPR addition in RONI & FPGA \\
\hline
\end{tabular}

Table 1. Required functions and proposed target architecture.

1) Central Processing Unit Operations (CPU) :

The Pre-processing part of the Reversible Watermarking is done in MATLAB.

Pre-processing-When image is received by the CPU from storage and followed by different operations like read image, conversion of image data, concatenations, cell to matrix operation.

Segmentation- Image will be partitioned into ROI (Region of Interest) and RONI (Region of Not Interest) using binary masking technique (roipoly). 
2) FPGA accelerator implementations (FPGA):

Securing the image - 16 bit secure key is embedded into the image for the security purpose is added in FPGA.

Watermarking with EPR- EPR is embedded as a watermark in the RONI of the image is added from FPGA programming.

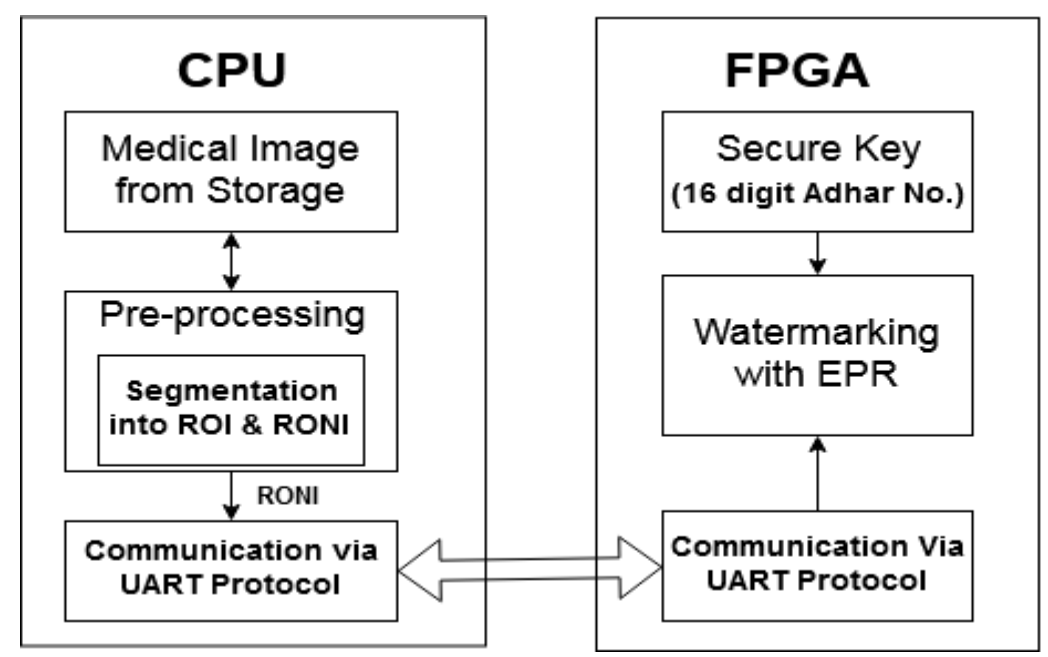

Fig. 1 Block Diagram of proposed Heterogeneous Architecture.

Reversible watermarking is implemented using integer transform based reversible contrast mapping algorithm will be used for partitioning of image into two, Region of Interest and Region of Non Interest. Medical image can be partitioned into non overlapping pixel groups in user defined space filling curve. Medical image is partitioned into $(32 \times 32)$ image block and pixel pairs are formed for further processing. For watermarking, embedding will be done using EPR (Electronic Patient Record) provided as a watermark is embedded into RONI of the image. Processed medical image with EPR will be watermarked using secure key. Watermarked image will be given to the output module. This watermarked image will appear on the display. This is the process of embedding watermark in the medical image.

3.1 Integer Transform based RCM Algorithm

As proposed by Coltuc et al., [1], lets $(0, \mathrm{~L})$ is gray level image and let $(p, q)$ are pixel pair. Forward transform of pixel,

$$
p^{\prime}=2 p-q, \quad q^{\prime}=2 q-p
$$

Transform restricted to sub-domain

$D \in[O, L] \times[O, L]$

Inverse transform of pixel

$$
p=\left(2 p^{\prime}+q^{\prime}\right) / 3, q=\left(p^{\prime}+2 q^{\prime}\right) / 3 .
$$

3.2 Watermark Embedding

The RCM algorithm used to implement reversible watermarking is shown in flowchart Fig. 2. 


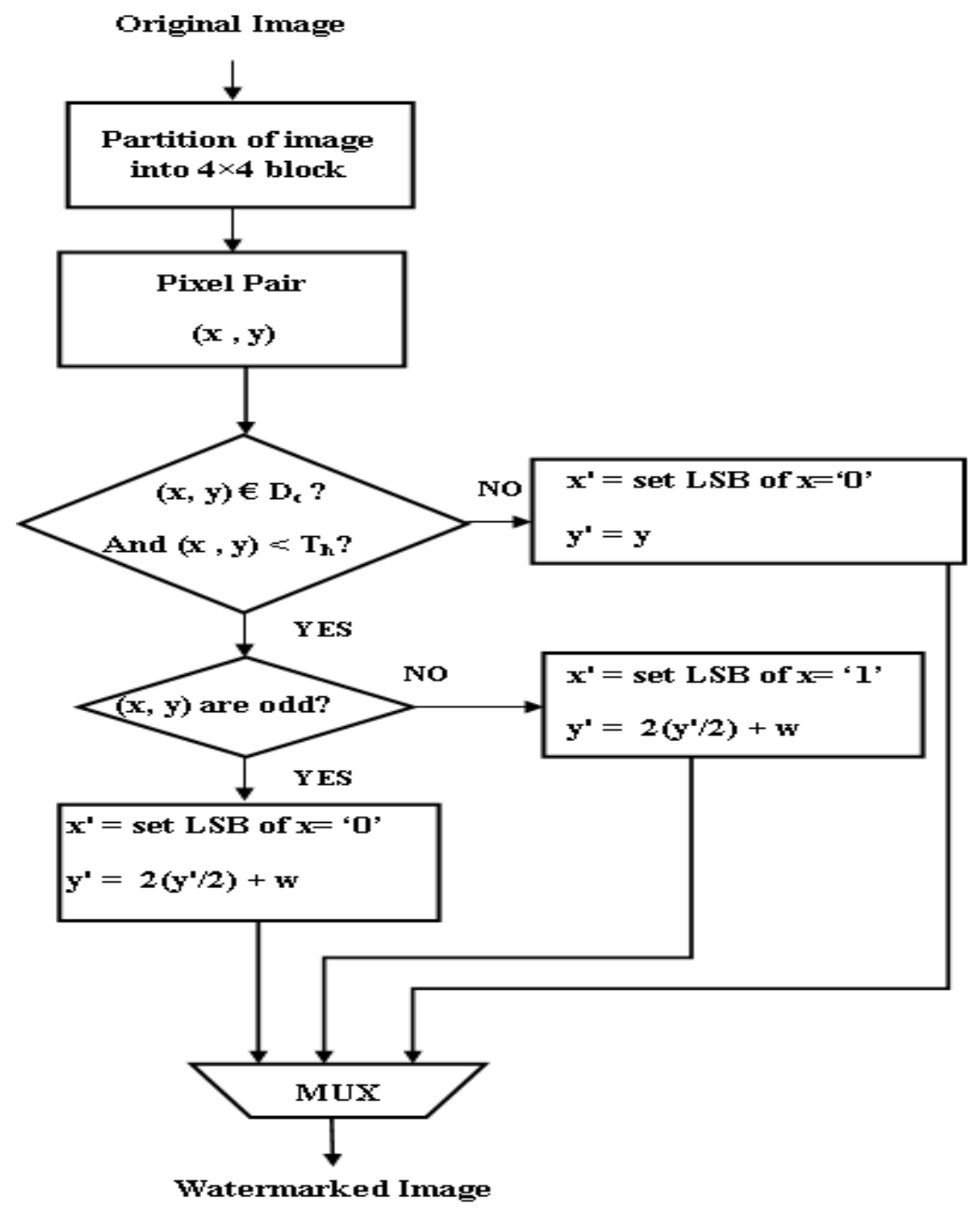

Fig. 2. Flowchart of proposed system using RCM

\section{FPGA Implementation}

Cyclone 4E FPGA is used in this implementation. Processed Medical Image is sent to FPGA kit using serial communication. USB cable is used for serial communication. Image is taken in the MATLAB converted to binary image and transmitted to FPGA kit using RS 232 cable, after reading the image in FPGA kit it is again transmitted from kit and displayed in MATLAB.

\subsection{Serial Communication using UART:}

Universal Asynchronous Receiver Transmitter protocol is used as communication standard. The data byte format is as shown in Fig. 3

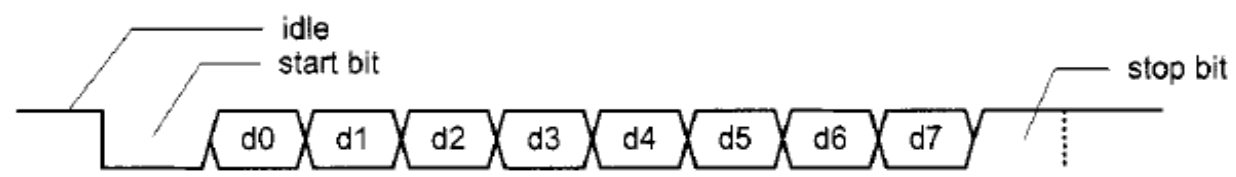

Fig. 3. UART bit Configuration

The baud rate used in the UART design are 9600, 11200, 115200 etc with the byte format as 8 data bits, 1 stop bit, and no parity bit. Complete implementation model for UART communication is shown in Fig.4 Baud generator, receiver and transmitter conditions are covered in this model 


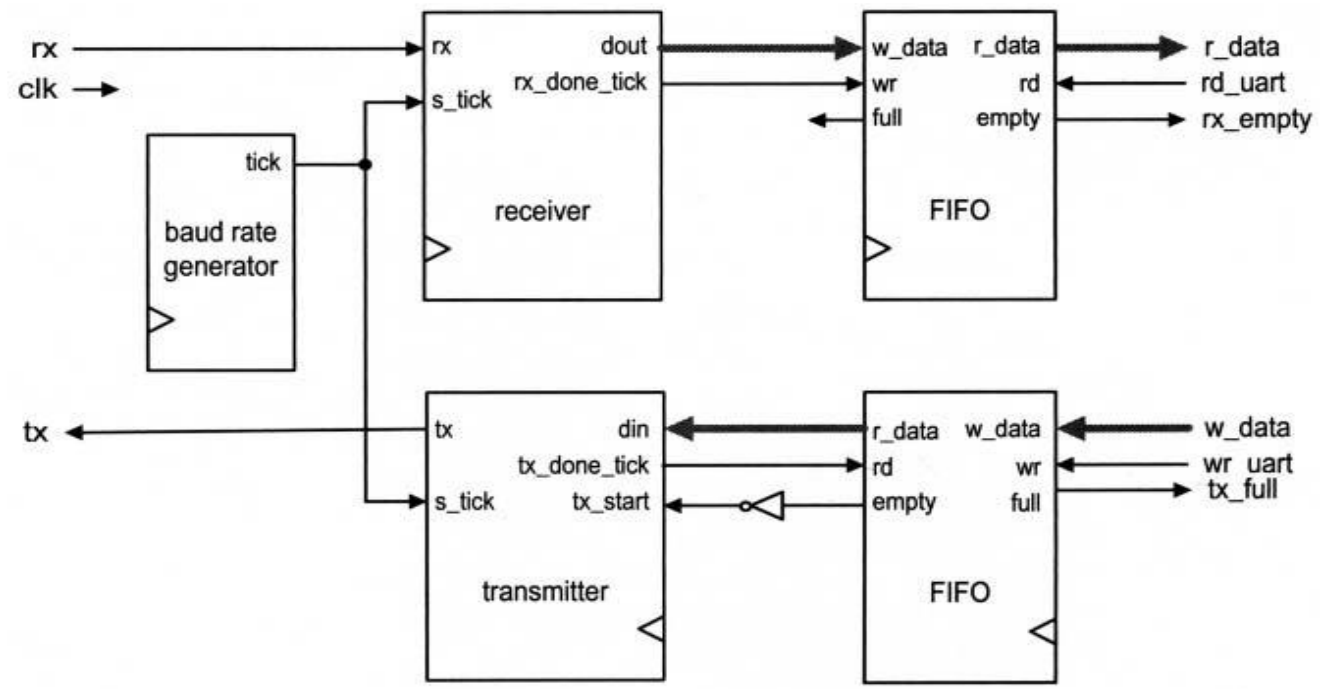

Fig.4 Complete UART System

\subsection{Baud Rate Generator Calculations}

For effective communication, 1,15, 200 baud rate is used. The sampling rate has to be 1,843,200 ticks per second by equation (4).

Baud rate generator $=16 * U A R T$ 's baud rate.

Since the FPGA kit used has a system clock rate of $50 \mathrm{MHz}$, the baud rate generator needs a mod-27, counter, in which the one-clock-cycle tick is asserted once every 27 clock cycles. Thus counter $=\left(50 \mathrm{MHz} /\left(16^{*} 1,15,200\right)\right)$.

\subsection{Simulation Results}

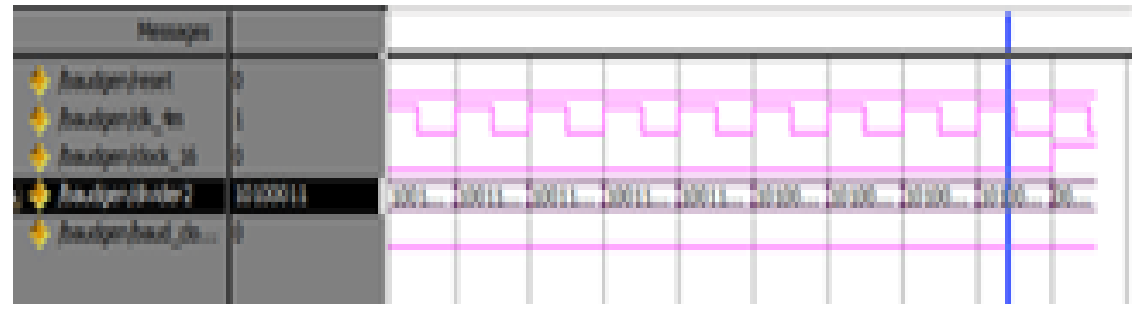

Fig. 5 Simulations for 115200 Baud Generator

For the UART communication between GUI and FPGA, RS 232 cable is used. For data transformation 1,15,200 baud rate is set. QUARTUS Pro software is used for coding and simulation results are seen in Fig. 5 shows the simulation result for baud rate generator, in which counter 27 is up counted.

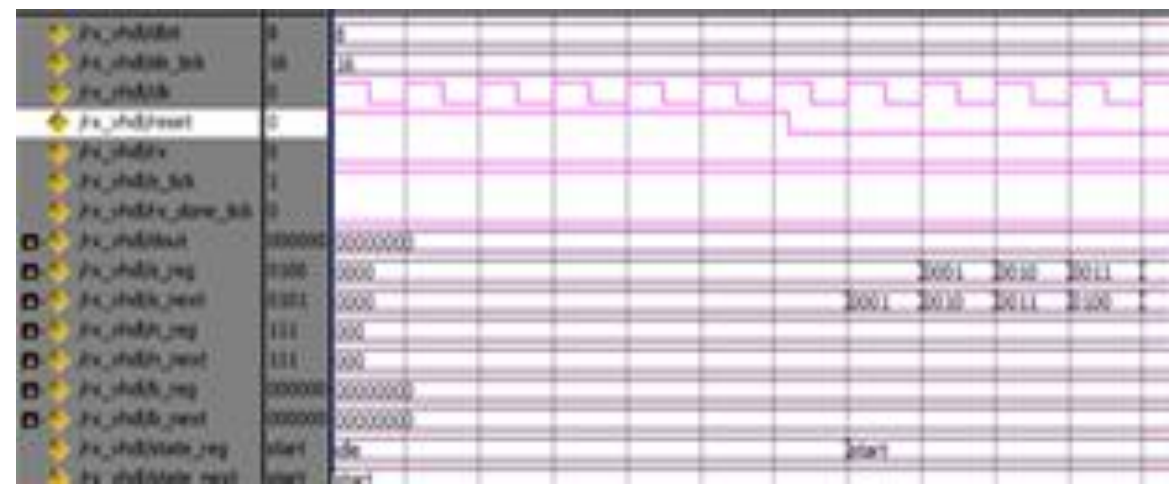

Fig. 6 Simulation for transmission

Fig. 6 shows the simulation result for data transmission. Conversion of data from idle to start can be seen in the waveform which indicates initialization of data transmission.

*Corresponding author: 


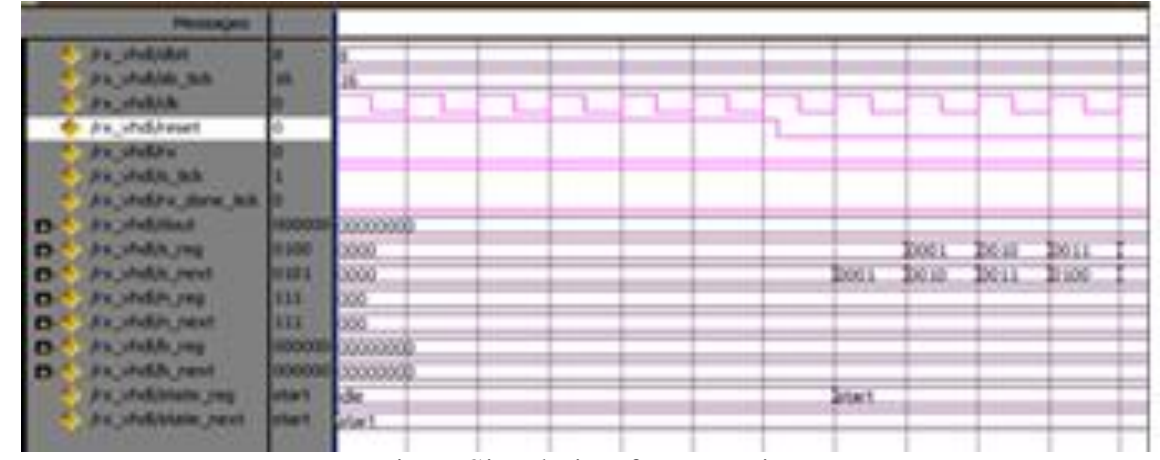

Fig. 7 Simulation for reception

Fig. 7 shows the simulation result for data reception. Conversion of data from idle to start can be seen in the waveform which indicates initialization of data reception.

\section{Results}

\subsection{FPGA Results}

RTL schematic is obtained after synthesizing all the Verilog codes required for Integer transform based RCM using UART communication on ALTERA DE2-115 kit using QUARTUS II software shown in Fig. 8 Device utilization summary required for hardware realization of integer transform based RCM on EP4CE115F29C7 Cyclone IV E FPGA is shown in Fig. 9

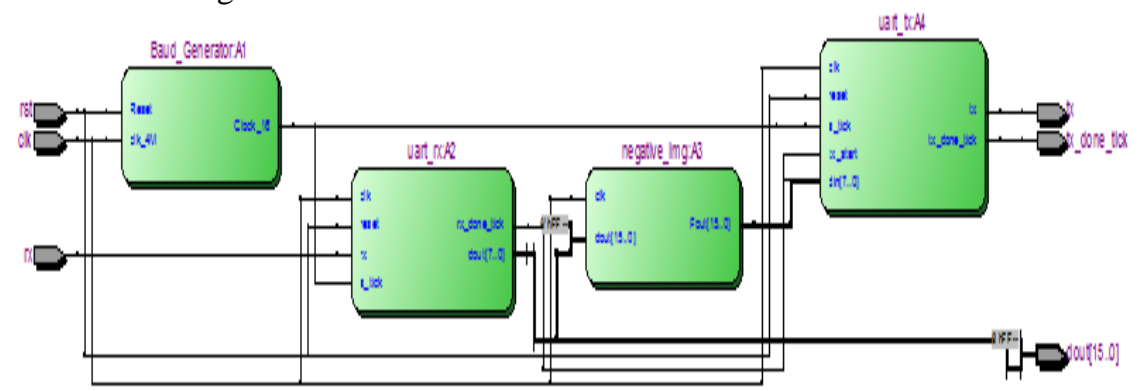

Fig.8 RTL Schematic of UART

\begin{tabular}{|c|c|}
\hline \multicolumn{2}{|l|}{ Gaverammany } \\
\hline Flow Seatas & Successt - Tue Fed 23 13:50:51 2018 \\
\hline Quertus II Version & 00.0 buld $21906 / 27 / 201053$ Web Edison \\
\hline Revioon Nome & UART_VERILG \\
\hline Toptevel Enbty Nome & uart_top \\
\hline Famly & Crdone WE \\
\hline Device & EACE115 $29 \mathrm{Cl}$ \\
\hline Tring Models & Find \\
\hline Met timing requrements & N/A \\
\hline - Total logic elenents & $94 / 114,400(<1 \%)$ \\
\hline Total combinatonal functions & $87 / 114,400(<1 \%)$ \\
\hline Dedcated logic registers & $52 / 114,430(<1 \%)$ \\
\hline Total regsters & $52+20$ \\
\hline Total pins & $21 / 529(4 \%)$ \\
\hline Tetal vitud pins & 0 \\
\hline Tetal menory bits & $0 / 3,981,312(0 \%)$ \\
\hline Enbedded Multolier 9 bit elenents & $0 / 532(0 \%)$ \\
\hline Totalpus & $0 / 4(0 \%)$ \\
\hline
\end{tabular}

Fig. 9 FPGA hardware summary

\subsection{Results for proposed heterogeneous architecture}

Different test images are taken from medical image datasets, and tested using Integer transform based RCM technique in MATLAB and in Quartus II ISE tool for hardware realization. DICOM image of brain is taken for study, the image was analysed using MATLAB. First of all image is partitioned into ROI and RONI. ROI is then embedded with the message bits. PSNR and SSIM are calculated for the watermarked image in Fig. 10 


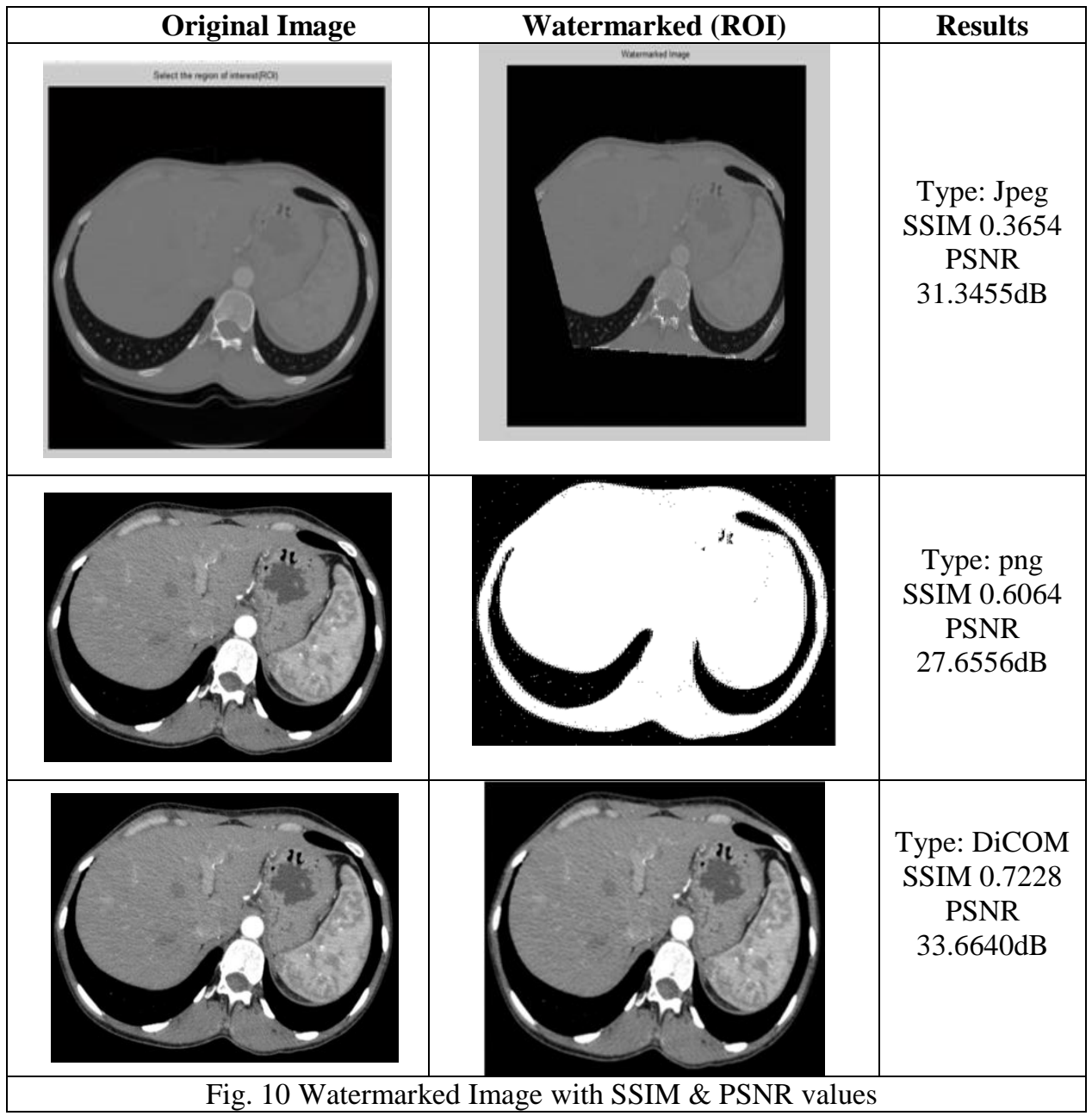

\section{CONCLUSION}

This paper proposes a FPGA based reversible watermarking of medical images using Integer transform based Reversible Contrast Mapping algorithm. Performance gain of this technique in terms of visual quality (PSNR) and imperceptibility (SSIM value) is analysed for medical images of brain. Hardware implementation using Altera DE2-115 kit on QUARTUS II software is simple, requires less area and power. Compare to other implementation like Hirak Maity et.al., [21] the results are better in terms of PSNR for jpeg Image. Compare to architecture of implementation, this design offers more flexibility in terms of heterogeneous design with two controllers offering execution data operations on Processor and execution signal operations on FPGA with fast computational time. Since the UART offer more communication overhead, can be reduced with other communication protocols.

\section{Ethics declarations}

Indicate all sources of financial support of each author and the team of authors.

This work was carried out under Ph.D. research program of corresponding author as research scholar under Visvesvaraya Technological University, Belagavi University. No financial sources are used.

\section{Indicate Conflict of interest}

The authors declare that they have no conflict of interest.

\section{Ethical approval}

This article does not contain any studies with human participants or animals performed by any of the authors.

\section{Informed consent}

None.

\section{REFERENCES}

Coltuc, D., Chassery, J.-M., 2007. Very fast watermarking by reversible contrast map-ping. IEEE Signal Process. Lett. 14 (April (4)), 255-258. 
Coltuc, D., Tudoroiu, A., 2012. Multibit versus multilevel embedding in high capacity difference expansion reversible watermarking. In: Proc. 20th European Signal Processing Conference (EUSIPCO 2012), Bucharest, Romania, pp. 1791-1795.

Coltuc, D., Tremeau, A., Delp, E.J., Wong, P.W., 2005. Simple reversible watermark-ing schemes. In: Proc. SPIE: Security, Steganography, Watermarking Multimedia Contents VII, Vol. 5681, pp. 561-568.

Coltuc, D., Chassery, J.-M., Delp, E.J., Wong, P.W., 2006. Simple reversible water-marking schemes: further results. In: Proc. SPIE: Security, Steganography, Watermarking Multimedia Contents VIII, Vol. 6072, pp. 739-746.

Coltuc, D., 2012. Low distortion transform for reversible watermarking. IEEE Trans.Image Process. 21 (1), $412-417$.

Dragoi, C., Coltuc, D., 2012. Improved rhombus interpolation for reversible water-marking by difference expansion. In: Proc. 20th European Signal Processing Conference (EUSIPCO 2012), Bucharest, Romania, pp. 1688-1692.

K. Matsui and K. Tanaka, "Video-steganography: How to secretly embed a signature in a picture," Proceedings IMA Intellectual Property Project, pp. 187-206, 1994.

Celik, M.U., Sharma, G., Tekalp, A.M., Saber, E., 2002. Reversible data hiding. In:Proceedings of the International Conference on Image Processing, NY, USA, pp.157-160.

Celik, M.U., Sharma, G., Tekalp, A.M., Saber, E., 2005. Lossless generalized-LSB dataembedding. IEEE Trans. Image Process. 14 (February (2)), 253-266.

Vleeschouwer, C.De., Delaigle, J.F., Macq, B., 2001. Circular interpretation of his-togram for reversible watermarking. In: Proceedings of the IEEE 4th Workshopon Multimedia Signal Processing, France, pp. $345-350$.

Vleeschouwer, C.De., Delaigle, J.F., Macq, B., 2003. Circular interpretation of bijectivetransformations in lossless watermarking for media asset management. IEEETrans. Multimed. 5 (March (1)), 97-105.

Weng, S., Zhao, Y., Pan, J.-S., Ni, R., 2008. Reversible watermarking based on invari-ability and adjustment on pixel pairs. IEEE Signal Process. Lett. 15, 721-724.

Weng, S., Pan, J.-S., Gao, X., 2012. Reversible watermark combining pre-processingoperation and histogram shifting. J. Inf. Hiding Multimed. Signal Process. 3(October (4)), 320-326.

Tian, J., 2003. Reversible data embedding using a difference expansion. IEEE Trans. Circuits Syst. Video Technol. 13 (August (8)), 890-896.

Weng, S., Zhao, Y., Pan, J.-S., Ni, R., 2008. Reversible watermarking based on invari-ability and adjustment on pixel pairs. IEEE Signal Process. Lett. 15, 721-724.

Wang, X., Li, X., Yang, B., Guo, Z., 2010. Efficient generalized integer transform for reversible watermarking. IEEE Signal Process. Lett. 17 (6), 567-570.

Dragoi, C., Coltuc, D., 2012. Improved rhombus interpolation for reversible water-marking by difference expansion. In: Proc. 20th European Signal Processing Conference (EUSIPCO 2012), Bucharest, Romania, pp. 1688-1692.

Hong, W., Chen, T.-S., Shiu, C.-W., 2009. Reversible data hiding for high qual-ity images using modification of prediction errors. J. Syst. Softw. 82 (11),1833-1842

MaityHK, MaitySP. Joint robust and reversible watermarking for medical images. Procedia technology 2012;6:275-282.

Zain JM, Baldwin LP, Clarke M. Reversible watermarking for authentication of DICOM images. In: Proceedings of the $26^{\text {th }}$ annual international conference of the IEEE Engineering in medicine and biology society, 2004.p.3237-3240.

Guo X, ZhuangTg. A region based lossless watermarking scheme for enhancing security of medical data. Journal of digital imaging 2009;

Kundu MK, Das S. Lossless ROI medical image watermarking technique with enhanced security and high payload embedding. In: Proceedings of the 20 th international conference on pattern recognition, Istanbul, Turkey; 2010.p.1457-1460.

Saraju Mohanty, N. Ranganathan and Ravi K. Namballa "VLSI implementation of invisible digital watermarking algorithms towards the developement of a secure jpeg encoder" Workshop on Signal Processing Systems, 2003.

Hirak Kumar Maity, Santi P. Maity, "FPGA implementation of reversible watermarking of the digital Image using reversible contrast mapping" The Journal of Systems and Software 2014.

Santi P. Maity $1 \cdot$ Hirak Kumar Maity, "On adaptive distortion control in reversible watermarking using modified reversible contrast mapping”, Springer Science Business Media New York, 2015. 